\title{
Comparison of electrostatic and electromagnetic synchronization of drift waves and suppression of drift wave turbulence in a linear device
}

\author{
C Brandt ${ }^{1}$, O Grulke Gr, $^{1,2}$ T Klinger ${ }^{1,2}$ \\ 1 Max-Planck-Institute for Plasma Physics, EURATOM Association, \\ Wendelsteinstraße 1, D-17491 Greifswald, Germany \\ 2 Ernst-Moritz-Arndt University, D-17489 Greifswald, Germany \\ E-mail: christian.brandt@lpmi.uhp-nancy.fr
}

\begin{abstract}
Experiments in a cylindrical magnetized plasma on the control of drift waves by means of two different spatiotemporal open-loop control systems - an electrostatic and an electromagnetic exciter - are reported. The drift wave dynamics is controlled by a mode-selective signal created with azimuthal arrangements of eight electrodes and eight saddle coils, respectively. Nonlinear interaction between the control signals and drift waves is observed, leading to synchronization of coherent drift waves and suppression of broadband drift wave turbulence. The cross-phase between density and potential fluctuations reduces from $\approx \pi / 2$ in turbulence to $\approx 0$ in controlled turbulence. Hence, the cross-field transport is reduced to the level of coherent drift waves. For both control systems the coupling to the drift wave can be ascribed to the drive of parallel currents, on the one hand via direct electric contact and on the other hand via electromagnetic induction.
\end{abstract}

PACS numbers: 05.45.Xt, 52.25.Xz, 52.30.Ex, 52.35.-g, 52.35.Kt, 52.35.Mw, 52.35.Ra

\section{Introduction}

In the magnetic confinement of hot plasmas a key problem is to reduce the turbulent cross-field transport of particles and energy. This so-called anomalous transport is by two orders of magnitude larger than collisional transport $[1,2,3]$. It is widely accepted that the drift wave instability drives turbulent transport in the plasma edge by phaseshifted density and potential fluctuations $[4,5,6,7]$. The drift wave instability is driven by the free energy of the density gradient and occurs in all bounded, magnetized plasmas. Changes of global parameters, like e.g. the plasma profile or the magnetic field geometry, have a strong influence on the drift wave dynamics. More subtle control methods are desired that leave average global plasma parameters and profiles constant. Relatively straightforward feedback techniques using electrodes immersed in the plasma have been shown to influence turbulence significantly $[8,9,10]$. Common to all those methods is the use of local electrodes, which produce a relatively large local perturbation of the 
plasma. Besides, electrode biasing is problematic for high-temperature plasmas because of rapid damage of the electrodes. A noninvasive approach for drift wave dynamics control is based on the use of external magnetic field coils. External magnetic field coils have already been applied in high-temperature plasmas to control the plasma edge transport, plasma rotation, magnetohydrodynamic instabilities and edge-localized modes $[11,12,13,14,15]$.

It is reasonable to complement the ongoing research in magnetic confinement devices with a dedicated study in a laboratory experiment with linear magnetic field geometry. This is not only to separate the physics mechanisms from effects arising from the magnetic field geometry but also because of the simple fact of better experimental access. In linear magnetic field geometry, open-loop control of drift wave dynamics has been extensively studied [16, 17, 18], exclusively using spatially distributed sets of electrodes in contact with the plasma. A single attempt to control drift waves with a set of external magnetic saddle coils has been made by Kauschke [19]; non-linear interaction between the control signal and the drift waves has been demonstrated. The physics mechanisms of the actual control process has not been fully understood for both cases.

In the present paper we study the dynamic control of coherent self-excited drift waves and drift wave turbulence by using two different open-loop control systems: Firstly, an octupole exciter with electrodes in contact with the plasma and secondly a set of eight hairpin saddle coils outside the plasma. The experimental device is the low- $\beta$ helicon device VINETA. The device and the characteristics of the self-excited drift waves are presented in Sec.2.1. The open-loop control systems as well as their influence on the plasma equilibrium are described in Sec. 2.2. Section 3 reports on the experiments to synchronize coherent drift waves and drift wave turbulence. The results are summarized and conclusions are drawn in Sec. 4.

\section{Experimental setup and characteristics of control systems}

\subsection{Drift waves in the experimental device VINETA}

The experiments were conducted in the linear, magnetized, low- $\beta$ plasma device VINETA [20]. The vacuum chamber, schematically depicted in Fig. 1, consists of a cylindrical stainless steel vessel with a radius of $0.2 \mathrm{~m}$ and a total length of $4.5 \mathrm{~m}$. The magnetic field is generated by 36 magnetic field coils, which establish a homogeneous magnetic field of $B_{0} \leq 100 \mathrm{mT}$. Argon plasma is produced by a helicon discharge with electron temperatures of $T_{e} \approx 2 \mathrm{eV}$, ion temperatures of $T_{i} \approx 0.25 \mathrm{eV}$ and plasma densities of $n_{e} \leq 1 \times 10^{19} \mathrm{~m}^{-3}$ in the center [21]. The argon ions have typically a gyro radius of $5 \mathrm{~mm}$ and are due to the high collisionality of the helicon plasma only weakly magnetized $\left(\omega_{c i} / \nu_{i n} \approx 1, \omega_{c i} / \nu_{i i} \ll 1, \omega_{c i} / \nu_{i e} \ll 1\right)$. For the probe diagnostics two positioning systems can be used, which provide radial or full azimuthal scans. The space-time structure of density fluctuations caused by drift waves is measured with an 


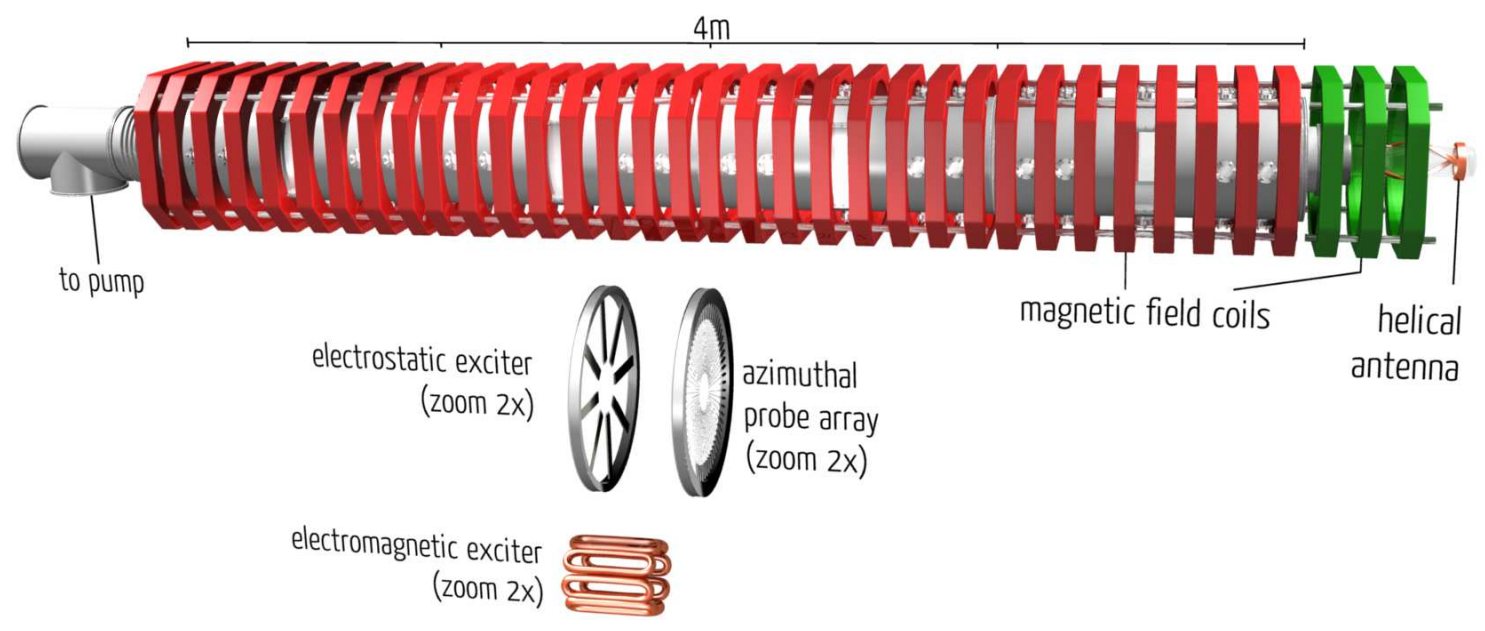

Figure 1. (Color online) Schematic drawing of the helicon plasma device VINETA. The respective size and position of the azimuthal probe array, the electrostatic, and electromagnetic exciter are depicted. The detailed views of the exciter are shown in Fig. 3

azimuthal probe array consisting of 64 Langmuir probes [22]. The azimuthal probe array, as well as the electrostatic and the electromagnetic exciter are schematically shown in Fig. 1.

The shape of the radial density profile of the helicon discharge is close to a Gaussian. In the region of the maximum density gradient fluctuations of density and potential due to the drift wave instability are observed, which propagate azimuthally in electron diamagnetic direction with typical frequencies between $f=1-20 \mathrm{kHz}$ being much smaller than the ion cyclotron frequency [23]. Coherent drift waves form azimuthal eigenmode structures. Figure 2 (a) shows the frequency spectrum of density fluctuations of a coherent $m_{0}=3$ mode peaking at $5.8 \mathrm{kHz}$ and its higher harmonics. The relatively
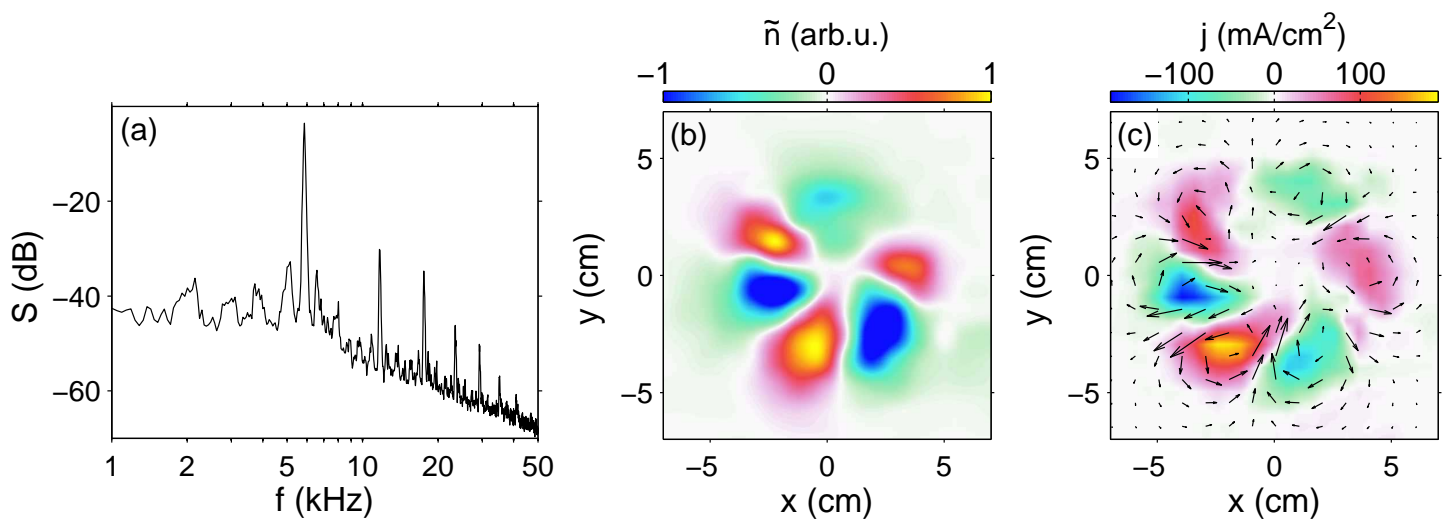

Figure 2. (Color online) A typical $m_{0}=3$ drift wave mode. (a) Frequency spectrum, (b) density mode structure and (c) parallel current filaments in the azimuthal plane. The arrows of the vector plot in (c) correspond to the magnitude of the local magnetic field. 
small diameter of the plasma column $(\approx 10 \mathrm{~cm})$ is not changing the fundamental properties and the dynamics of the drift wave as the radial extend of the modes is still smaller than the radius of the plasma column [23, 24]. The spatial mode structure of the density fluctuations in the azimuthal plane is depicted in Fig. 2(b). The maximum mode amplitude is located at the maximum density gradient which peaks at $r \approx 3 \mathrm{~cm}$. In the plasma center the mode structure disappears. A main characteristic of drift wave dynamics are the associated parallel electron currents [6]. Figure 2(c) depicts the parallel current density in the azimuthal plane as reconstructed by measurements of the associated magnetic field fluctuations with $\dot{B}$-probes at the same time instant as the density fluctuations. The pattern of the current filaments corresponds to the density mode structure and hence confirms the tight coupling between the parallel electron dynamics and the $E \times B$-convection perpendicular to the magnetic field [25]. Figure 2 clearly shows three current filaments in positive and three in negative $z$-direction. The magnitude of the current density is $j \approx 100 \mathrm{~mA} / \mathrm{cm}^{2}$.

\subsection{Exciter schemes}

Two different control schemes are applied to control the dynamics of drift waves. Figure $3(a, b)$ shows the setup of the electrostatic octupole exciter. It consists of eight stainlesssteel electrodes, which are azimuthally arranged on the plasma circumference. Each electrode has a size of $5 \times 20 \mathrm{~mm}$ and is introduced into the plasma at a radial position $r=4.2 \mathrm{~cm}$. The photo of the exciter [Fig. 3(b)] also depicts the mounting and the electric connections to the electrodes. The electrodes are connected to audio power amplifiers with a linear frequency response in the range of $f=1-20 \mathrm{kHz}$ providing

(a)

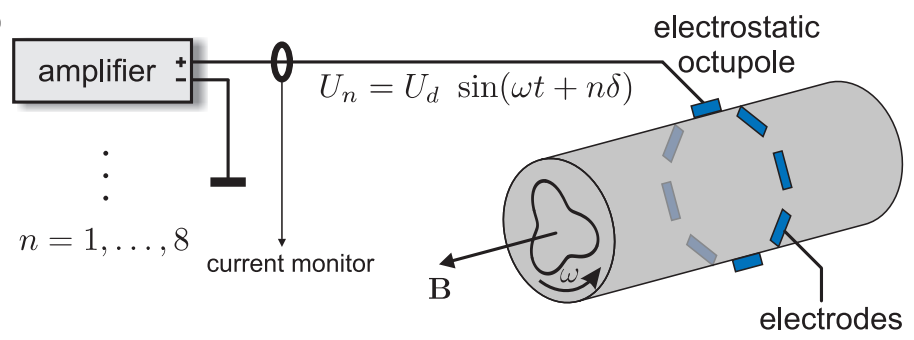

(c)

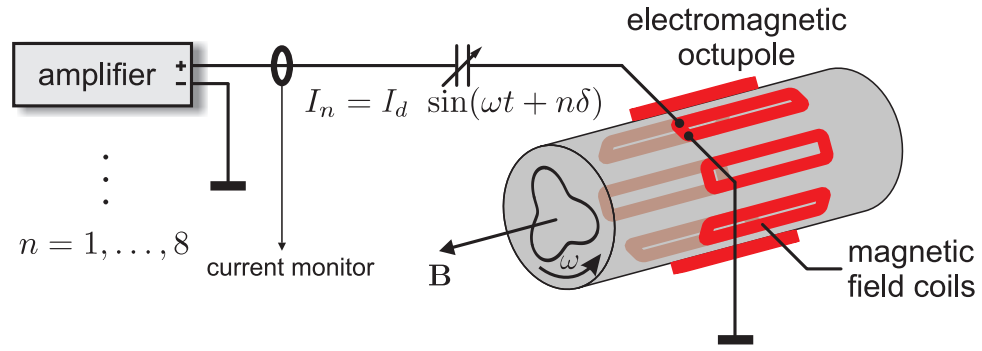

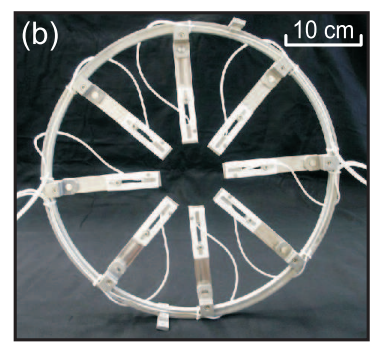

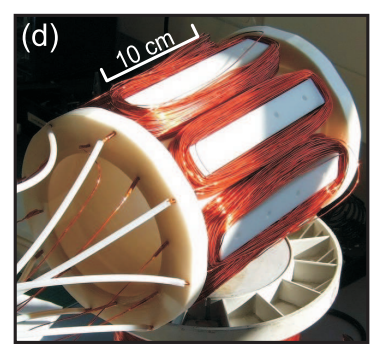

Figure 3. (Color online) Schematic setup and picture of the electrostatic exciter $(a, b)$ and of the electromagnetic exciter $(c, d)$. 
plasma currents up to $I_{d}=20 \mathrm{~A}$ per electrode corresponding to a current density of $j \approx 10000 \mathrm{~mA} / \mathrm{cm}^{2}$. A set of current monitors for each electrode is used to observe the driven current. The electrodes are driven with sinusoidal voltages of the form

$$
U_{n}(t)=U_{d} \sin \left(2 \pi f_{d} t+n \delta\right) \text {. }
$$

The phase angle between each electrode pair $(n, n+1)$ is set to

$$
\delta= \pm \frac{2 \pi m_{d}}{k}
$$

with $m_{d}$ being the mode number of the exciter signal and $k$ the number of electrodes $(k=8)$. Given by the number of electrodes, the maximum mode number of the control is limited to $m_{d} \leq 3$ owing to the Nyquist-limit $\left(m_{d}<k / 2\right)$. The sign of the phase shift defines the propagation direction of the spatiotemporal control signal (co- or counterrotating with respect to the drift mode propagation).

The setup of the second control system, the electromagnetic octupole exciter, is shown in Fig. 3(c,d). It consists of eight saddle coils, azimuthally arranged around the center of the plasma column at a radius of $6 \mathrm{~cm}$. Each coil has 100 windings and is connected to the same high power amplifiers as the electrostatic exciter. An alternating current is applied to the coils

$$
I_{n}(t)=I_{d} \sin \left(2 \pi f_{d} t+n \delta\right),
$$

with a phase shift

$$
\delta= \pm \frac{2 \pi m_{d}}{k}
$$

similar to the electrostatic exciter. The mode number is restricted by the Nyquist-limit to $m_{d} \leq 3$ also in this case. The coils are operated in series resonance circuits to account to the coil inductance. By using variable capacitors, the circuits are impedance matched for frequencies in the range of $f=0.9-14 \mathrm{~Hz}$.

Both exciter schemes generate azimuthally propagating current filament mode patterns matching the mode structures of drift waves [cf. Fig. 2]. The propagation velocity of the patterns can be varied via the frequency $f_{d}$. Given by the potential difference between the electrodes of the electrostatic exciter and the plasma potential, parallel currents are drawn. For the measurement of the driven plasma currents, the azimuthal plane was scanned with a sensitive B-dot probe [26]. To distinguish between the driven current filaments and current fluctuations caused by drift wave activities a measurement was performed under plasma conditions without drift wave activities. Figure 4(a-c) shows magnetic field fluctuations as vector plots and the associated current filaments in the azimuthal plane for mode numbers $m_{d}=1,2,3$. The length of each arrow corresponds to the local magnitude of the magnetic field and the black bars indicate the positions of the electrodes. Parallel current filaments are driven roughly at the electrode position. The current pattern propagates in the predefined direction. In each case there are $m_{d}$ filaments with positive and negative current, i.e. along and opposite to the direction of the ambient magnetic field. By comparing these driven current density patterns with the current density patterns of the drift waves [cf. Fig. 

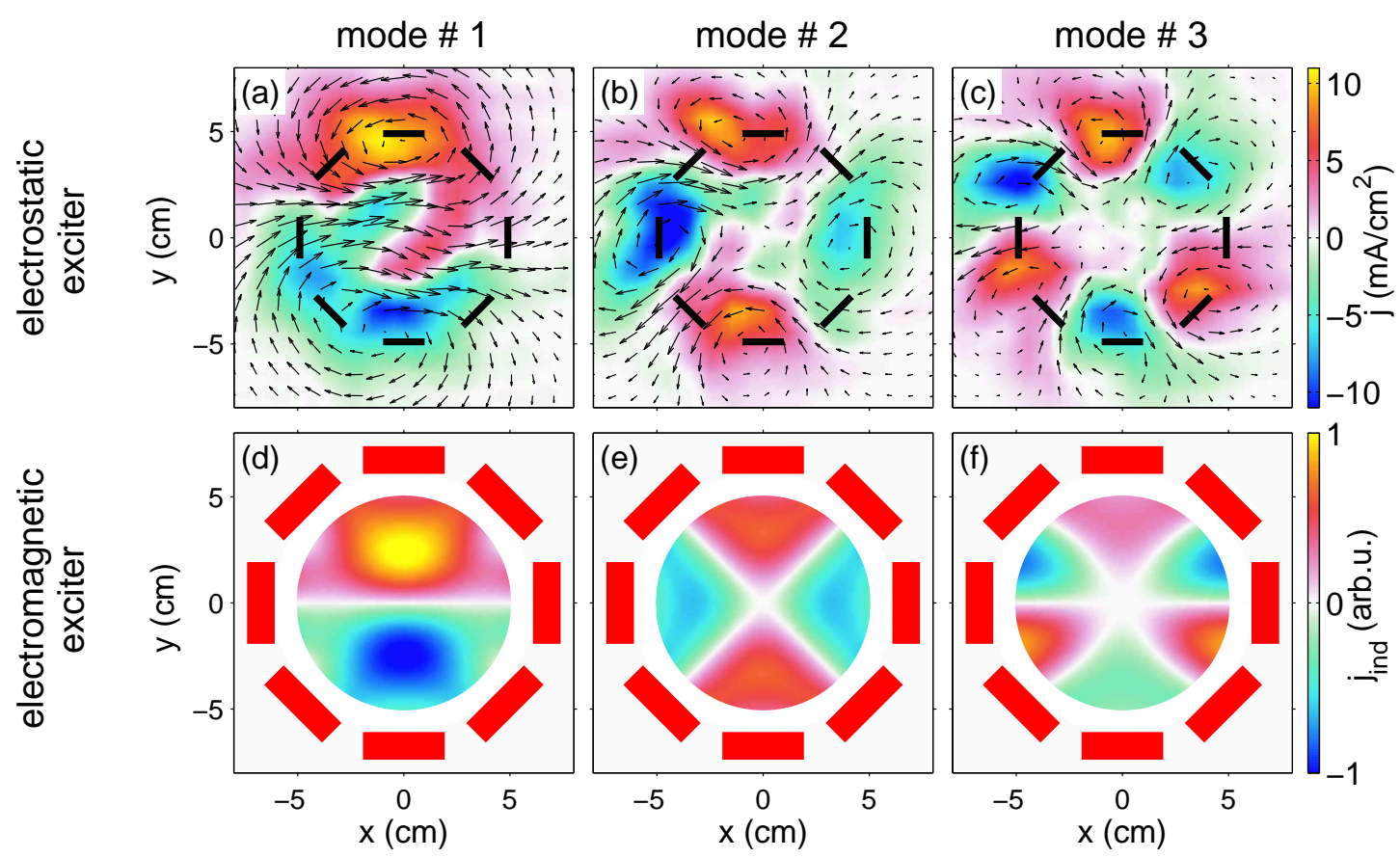

Figure 4. (Color online) Mode selective drive of parallel currents with the electrostatic and electromagnetic exciter for mode numbers $m_{d}=1,2,3$. (a-c) Measurement of the parallel currents driven by the electrostatic exciter. The vectors correspond to the measured magnetic field $B_{x y}$ in the azimuthal plane. The position of the exciter electrodes are indicated by black bars. (d-f) Calculation of the induced parallel current pattern into the plasma by the electromagnetic exciter. The position of the magnetic field coils are indicated by red bars.

2 ], it is evident that the exciter currents are very similar to the spatial structure of the intrinsic drift wave currents. In the present case the amplitude of the driven current density [Fig. 4] is relatively small with $j_{d} \approx 10 \mathrm{~mA} / \mathrm{cm}^{2}$ due to the low plasma density and the smaller potential applied to the electrodes. In the maximum density regime and high exciter amplitudes, the exciter current density can be up to $2000 \mathrm{~mA} / \mathrm{cm}^{2}$.

The electromagnetic exciter is a contactless control system using external magnetic field coils. The temporal variation of the magnetic exciter field induces currents into the plasma and the induction currents flow along the ambient magnetic field. Hence, only the currents in the parallel coil wires induce significant plasma currents. For a quantitative estimate of the induced parallel plasma currents, a simplified model is applied, where only the parallel wires of the exciter are taken into account and are assumed as thin wires. The induced current at a position $(x, y)$ is proportional to the temporal change of the magnetic exciter field $\partial B(x, y) / \partial t$. The contribution of each parallel coil section is summed up for each position in the azimuthal plane to obtain the space-time structure of the magnetic exciter field. The induced current is proportional to the plasma electron density. A Gaussian shaped density profile $n(x, y)$ is assumed, typical for the helicon discharge. Using the magnetic field of a long thin 
wire $B_{n}= \pm \mu_{r} \mu_{0} I_{d} \sin \left(\omega_{d} t+q \delta\right) /(2 \pi r)(n=1 \ldots 8)$ the induced current finally reads

$$
j_{i n d}(x, y) \sim \partial_{t} B(x, y) \cdot n(x, y) \sim \omega_{d} \sum_{n=1}^{8} \sum_{i=1}^{2}(-1)^{i} \frac{\cos \left(\omega_{d} t+q \delta\right)}{d_{n, i}} \cdot n(x, y),
$$

with $d_{n, i}$ being the distance between the wire $i$ of coil $n$ and the spatial point in the $(x, y)$-plane. Figure 4(d-f) depicts the calculated induced parallel plasma currents in the azimuthal plane for the mode numbers $m_{d}=1,2,3$. The induced plasma current amplitude is obtained by considering the Spitzer resistivity

$$
\eta=\frac{8}{3 \sqrt{3}} \frac{\ln \Lambda\left(\pi e^{2} m_{e}^{1 / 2}\right)}{\left(4 \pi \varepsilon_{0}\right)^{2} \cdot(k T)^{3 / 2}} .
$$

For the present low temperature plasma situation one obtains $\eta=5.3 \cdot 10^{-4} \Omega \mathrm{m}$ (for $T_{e}=2 \mathrm{eV}, \ln \Lambda \approx 10$ ) at a distance $d=5 \mathrm{~cm}$ away from the coil. Further, an exciter frequency of $f_{d}=5 \mathrm{kHz}$, a maximum exciter $B_{x y}$-amplitude of $B_{d}=0.1 \mathrm{mT}$, and a coil length of $l=14 \mathrm{~cm}$ are taken as parameters. In this case $j=l \omega_{d} B_{d} / \eta$ yields a maximum current density of $j_{d} \approx 82 \mathrm{~mA} / \mathrm{cm}^{2}$, which is similar to the current density of $j_{0} \approx 100 \mathrm{~mA} / \mathrm{cm}^{2}$ as observed for drift waves.

\section{Control of coherent drift modes and drift wave turbulence}

The direction of the exciter pattern is called "co-rotating" if it propagates parallel to the phase fronts of the drift waves. The opposite direction is called "counter-rotating".

\subsection{Electrostatic synchronization}

For the control experiments, the electrostatic exciter is installed in the vessel of the VINETA device at the position indicated in Fig. 1. Firstly the control of coherent drift modes is discussed, followed by the control of drift wave turbulence.

3.1.1. Drift modes Figure 5 shows the frequency spectrum and the space-time diagram of a single coherent $m_{0}=1$ drift mode. The frequency of the drift wave corresponds to the dominant peak at $f_{0}=3.3 \mathrm{kHz}$. The peaks at $2 f_{0}, 3 f_{0}$ and $4 f_{0}$ are the higher harmonics. To synchronize the drift wave, the electrostatic exciter is operated with
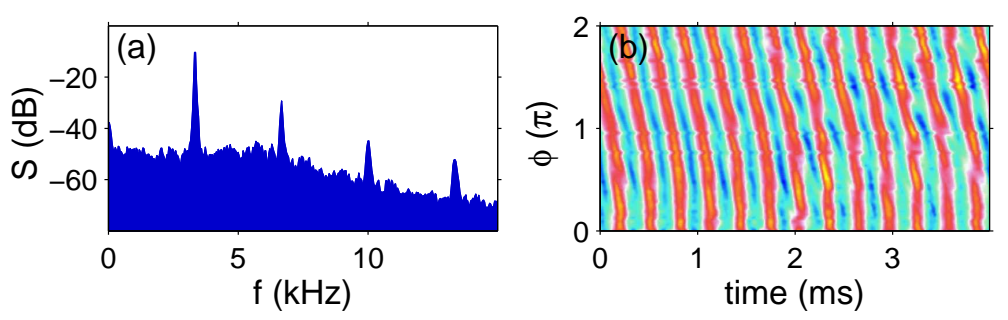

Figure 5. (Color online) Frequency spectrum (a) and space-time diagram (b) of a coherent $m_{0}=1$ drift wave mode. 

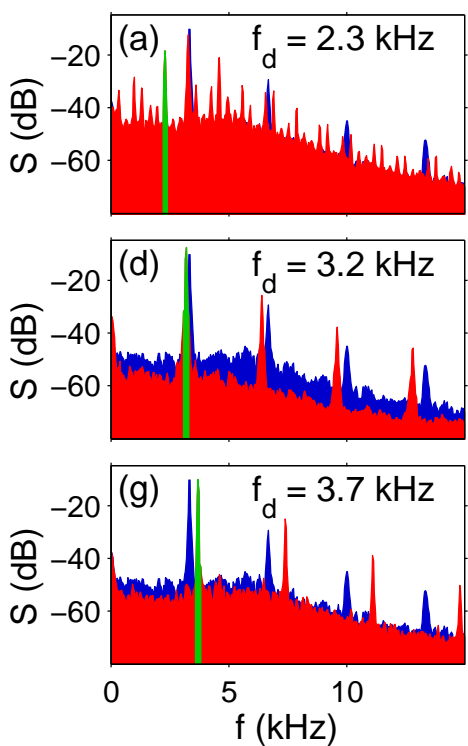
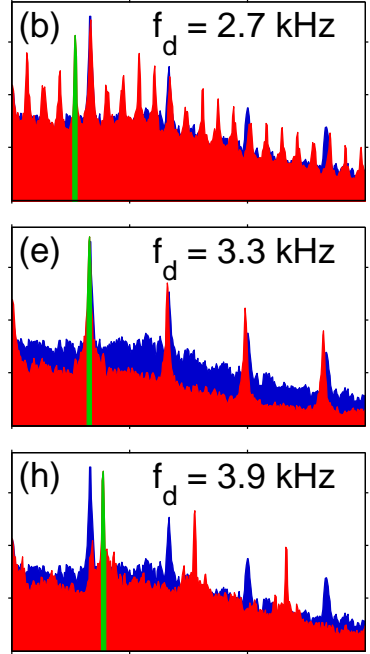

${ }^{5}(\mathrm{kHz})$
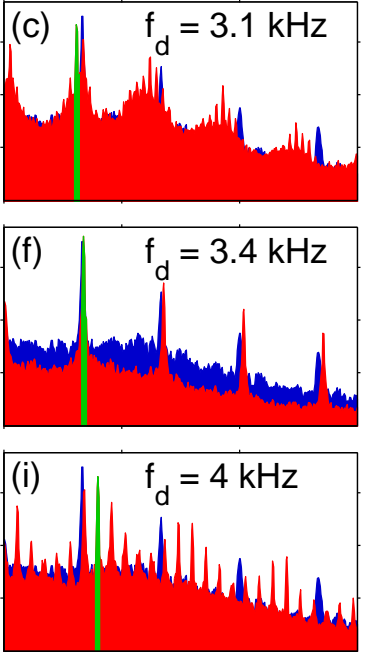

0

$\mathrm{f}(\mathrm{kHz})$

Figure 6. (Color online) Synchronization of a coherent $m_{0}=1$ drift wave mode. The sequence of frequency spectra are shown, where the exciter frequency is scanned from $2.3 \mathrm{kHz}$ to $4.0 \mathrm{kHz}$. The spectrum of the coherent drift wave is superimposed in blue. (a-c) $f_{d}<f_{0}$ no synchronization, (d-h) synchronized drift wave, (i) drift wave unlocks.

a co-rotating $m_{d}=1$ mode pattern. In consecutive discharges the exciter frequency is scanned across the drift wave frequency over the range $f_{d}=2.3-4.3 \mathrm{kHz}$. During the frequency scan, the exciter amplitude is kept constant at voltage $U_{d}=25 \mathrm{~V}$, corresponding to a current density $j_{d}=79 \mathrm{~mA} / \mathrm{cm}^{2}$. Figure 6 shows the results of the frequency scan as a sequence of frequency spectra. The spectrum of the drift wave [Fig. 5] is underlayed in blue for reference. Additionally, the exciter frequency is marked with a green bar for each spectrum. The first three diagrams [Fig. 6(a-c)] show the exciter frequency $f_{d}$ approaching the drift wave frequency $f_{0}$. Although the drift wave frequency is not changed, the appearance of sidebands in the spectrum indicates nonlinear interaction between the exciter signal and the drift wave. Each sideband corresponds to the sum or difference between the drift wave frequency and the exciter frequency or integer multiples $p f_{d} \pm q f_{0}(p, q= \pm 1, \pm 2, \ldots)$. In Fig. 6(c) the exciter frequency $f_{d}$ is only $200 \mathrm{~Hz}$ smaller than the drift wave frequency $f_{0}$, resulting in a dense sideband structure with $\Delta f=200 \mathrm{~Hz}$ in the vicinity of the drift wave peak and its harmonics. The triangular shape of sideband groups is an indicator for periodic pulling $[27,28]$. In Figs. $6(\mathrm{~d}-\mathrm{h})$ the exciter frequency $f_{d}$ is sufficiently close to the drift wave frequency such that mode locking occurs. The drift wave is locked to the exciter signal and all sidebands vanish. Frequency pulling is found for the range $f=3.2-3.9 \mathrm{kHz}$. At $f_{d}=4.0 \mathrm{kHz}$ [Fig. 6(i)] the exciter frequency is too high for synchronization, the drift wave unlocks and the sidebands reappear.

Figure 7 shows the ion saturation current, the frequency spectrum, and the spacetime diagram of a synchronization experiment of a $m_{0}=3$ drift wave mode. The first row shows the drift wave peaking at $f_{0}=6 \mathrm{kHz}$, the second row shows the controlled 

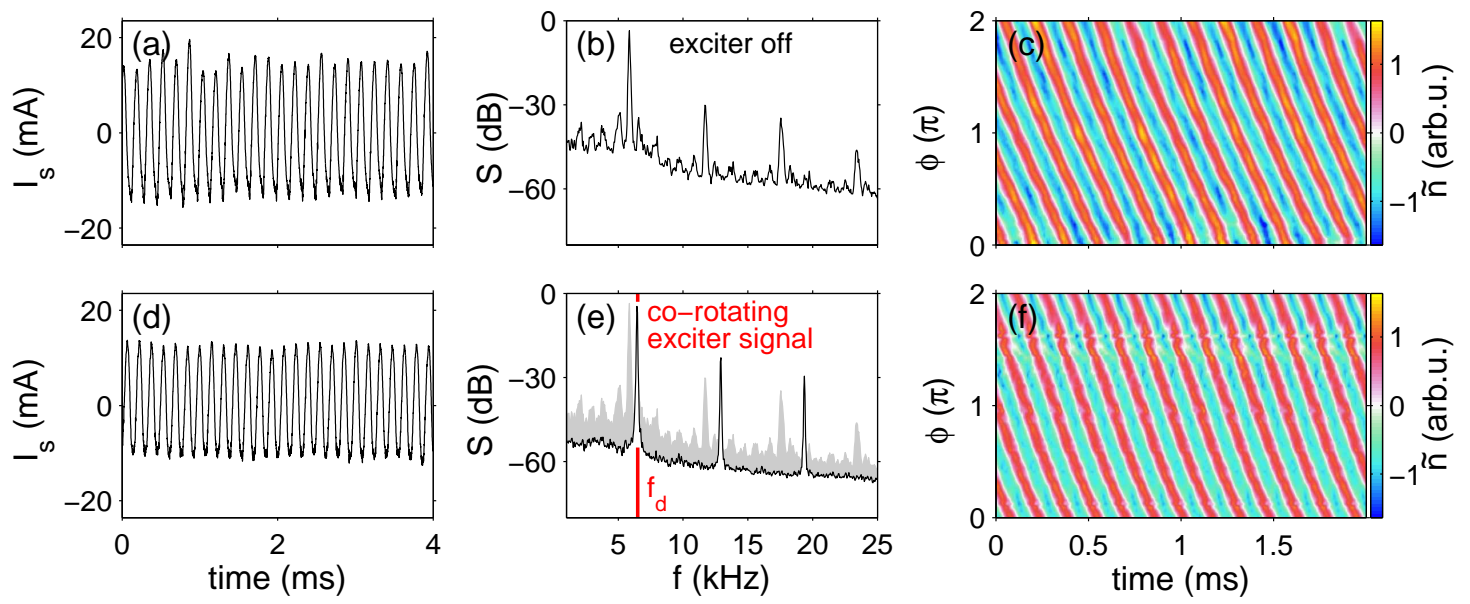

Figure 7. (Color online) Synchronization of a coherent $m_{0}=3$ drift wave with the electrostatic exciter with conducting electrodes. Columnwise are shown the ion saturation current, frequency spectrum and the space time diagram of the (a-c) unperturbed coherent $m_{0}=3 \mathrm{drift}$ wave mode at $f_{0}=6 \mathrm{kHz}$ and the (d-f) synchronized drift wave to the exciter mode number $m_{d}=3$ and exciter frequency $f_{d}=6.5 \mathrm{kHz}$. The amplitude of the exciter potential perturbation is $24 \mathrm{~V}$. The spectrum of Fig. (b) is highlighted in Fig. (e) as a grey background.
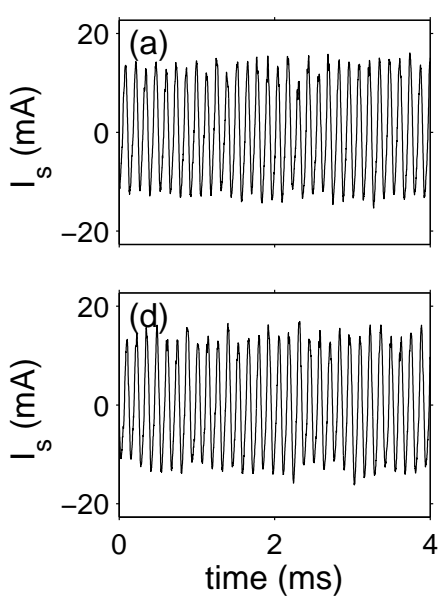
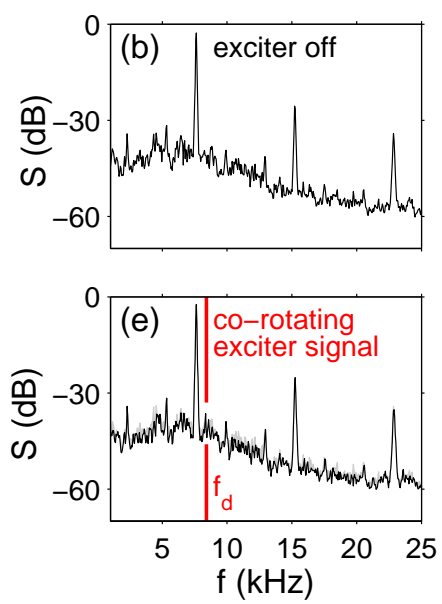
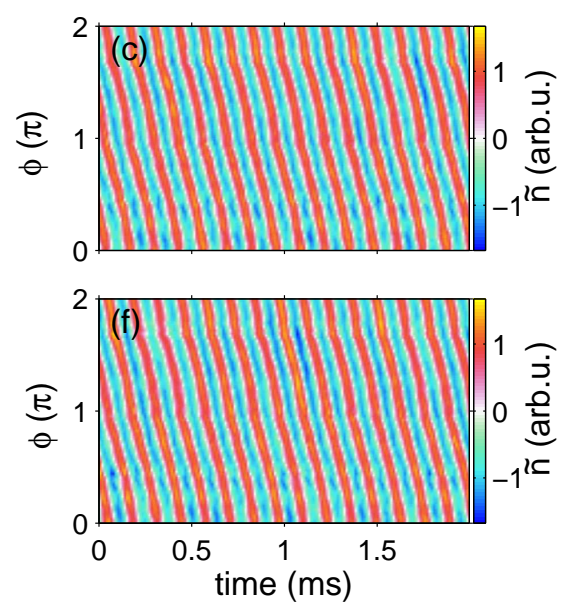

Figure 8. (Color online) No control effect on a coherent $m_{0}=2$ drift wave at $f_{0}=7.6 \mathrm{kHz}$ is observed applying the electrostatic exciter with insulated electrodes with an $m_{d}=2$ pattern and a frequency $f_{d}=8.4 \mathrm{kHz}$. Column by column the ion saturation current, the frequency spectrum, and the space-time diagram is shown for the drift wave (a-c) and for a co-rotating (d-f) exciter pattern with a voltage amplitude of $U_{d}=58 \mathrm{~V}$.

drift wave. The drift wave is synchronized by a $m_{d}=3$ exciter signal and a driver voltage $24 \mathrm{~V}$ at a frequency $f_{d}=6.5 \mathrm{kHz}$. The frequency spectrum in Fig. $7(\mathrm{e})$ clearly demonstrates locking of the drift wave to the exciter frequency and a reduction of the broadband spectral background. The space-time diagrams [Fig. $7(\mathrm{c}, \mathrm{f})]$ show that the mode number $m=3$ remains unchanged. The increased slope of the phase fronts in 
Fig. 7(f) indicates a slightly increased phase velocity due to the increased frequency.

During the two synchronization experiments above, the electrodes have been in direct contact with the plasma. Consequently the exciter drives potential perturbations as well as parallel plasma currents. These two effects are separated by avoiding the drive of plasma currents using electrically insulated electrodes. The result of the synchronization experiment is shown in Fig. 8. The arrangement of the figure is the same as in Fig. 7 . The first row shows a $m_{0}=2$ drift wave mode peaking at a frequency $f_{0}=7.6 \mathrm{kHz}$. The second row shows the attempt of synchronizing the mode by a corotating $m_{d}=2$ exciter signal with a frequency $f_{d}=8.4 \mathrm{kHz}$. A comparison of the frequency spectra of the unperturbed drift wave [Fig. 8(b)] and the drift wave with the co-rotating (but insulated) exciter [Fig. 8(e)] shows no change at all. Neither a distinct peak at the exciter frequency nor sidebands due to nonlinear interaction between exciter signal and the drift wave occur. The space-time pattern of the drift wave remains unchanged as well [Fig. 8(c,f)]. The drift wave is not controlled by the exciter signal, although the exciter is operated with a relatively high amplitude of $U_{d}=58 \mathrm{~V}$. This result clearly demonstrates the importance of the driven currents in the drift wave control mechanism.

3.1.2. Drift wave turbulence In drift wave turbulence a large number of drift modes are simultaneously present and the Fourier frequency spectrum is broadband. Fig. 9 shows the control of weakly developed drift wave turbulence, obtained with the electrostatic exciter. The first row [Fig. 9(a-d)] shows the uncontrolled, weakly developed drift wave turbulence. The ion saturation current fluctuations have an irregular character and the frequency spectrum has a power-law decrease with a spectral index $\alpha=-3.2$. In the space-time diagram, short- and long-lived structures of different spatial scales are observed. They predominantly propagate into the electron diamagnetic drift direction. The mode number-frequency spectrum is spread out in frequency and mode number space. In the second and third row the electrostatic exciter signals are applied with co- and counter-rotating patterns, respectively. The exciter operates with a $m_{d}=2$ mode pattern at a driver frequency $f_{d}=7 \mathrm{kHz}$. For the co-rotating exciter case a strong change of the drift wave dynamics is achieved [Fig. 9(e-h)]. The fluctuation signal is much more coherent and the frequency spectrum is peaked [Fig. 9(f)] with a reduced broad-band background. If the exciter signal is counter-rotating, the weakly developed turbulence remains essentially unchanged [Fig. 9(i-1)]. The fluctuation signal is incoherent and the frequency spectrum is similar to the uncontrolled plasma [Fig. 9 (a)], except for a small peak at the exciter frequency. This small influence is also seen in the space-time diagram [Fig. 9(k)] and in the mode number-frequency spectrum [Fig. $9(\mathrm{l})$ ], here with the occurrence of a $m=6$ mode. Responsible for this is the ambiguity of the exciter signal resulting from the limited number of electrodes $(k=8)$. Due to the Nyquist-limit a counter-rotating $m=2$ mode contains also a co-rotating $m=6$ mode pattern (corresponding to $k-2$ ). 

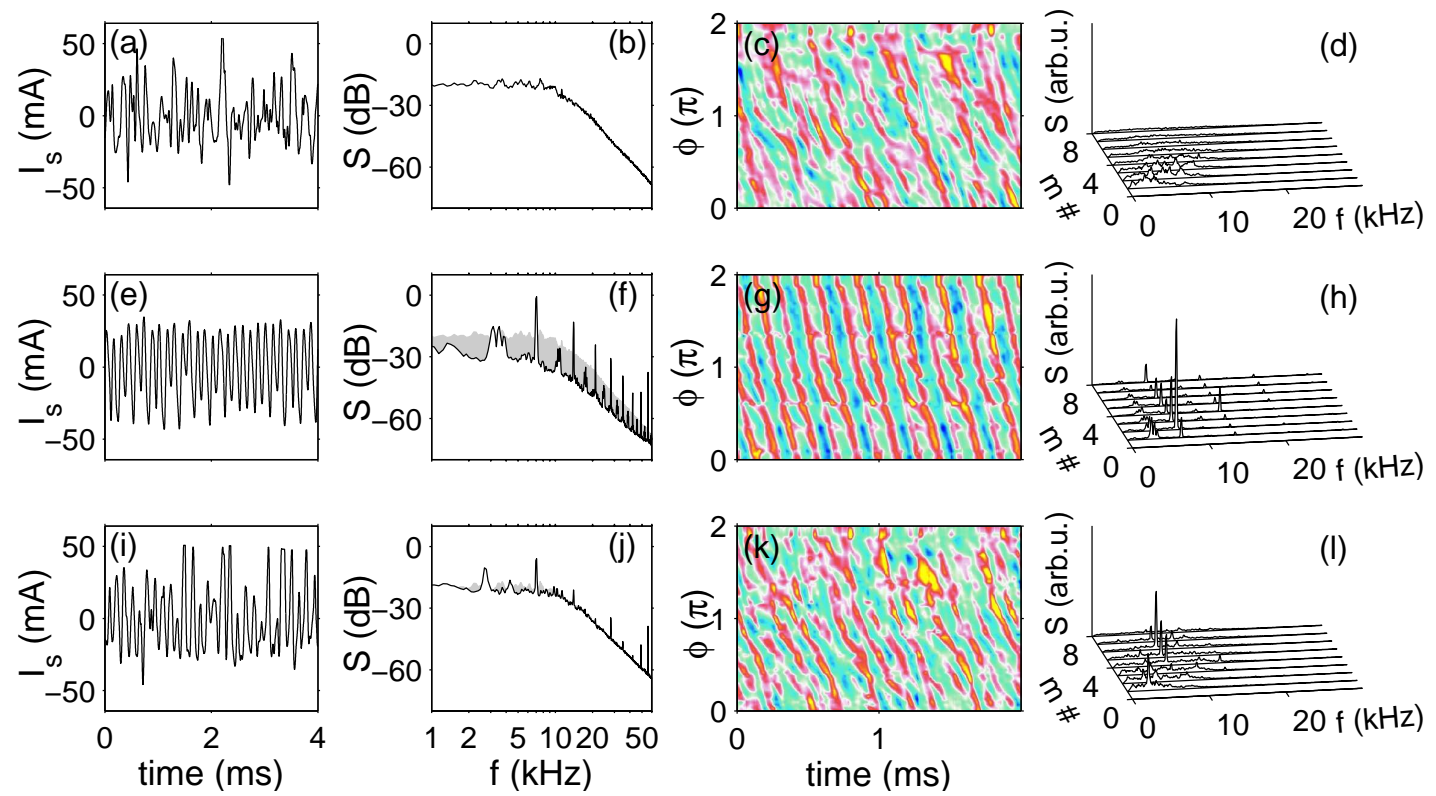

Figure 9. (Color online) Spatiotemporal control of weakly developed drift wave turbulence. Column by column the ion saturation currents, power spectra, spacetime diagrams, and mode number-frequency spectra are shown. The first row (a-d) represents weakly developed drift wave turbulence without control signal. The next rows show the controlled drift wave by the electrostatic exciter: (e-h) co-rotating, (il) counter-rotating. The exciter is operated with a $m_{d}=2$ mode and a frequency $f_{d}=7 \mathrm{kHz}$. The amplitude of the drawn current density is $j_{d}=480 \mathrm{~mA} / \mathrm{cm}^{2}$ for the co- and $300 \mathrm{~mA} / \mathrm{cm}^{2}$ for the counter-rotating exciter.

3.1.3. Influence on cross-field transport Figure 10 makes a comparison between uncontrolled [Fig. 10(a,b)] and controlled drift wave turbulence [Fig. 10(c,d)]. Shown are the cross-power spectra and the cross-phase spectra of density and potential fluctuations.
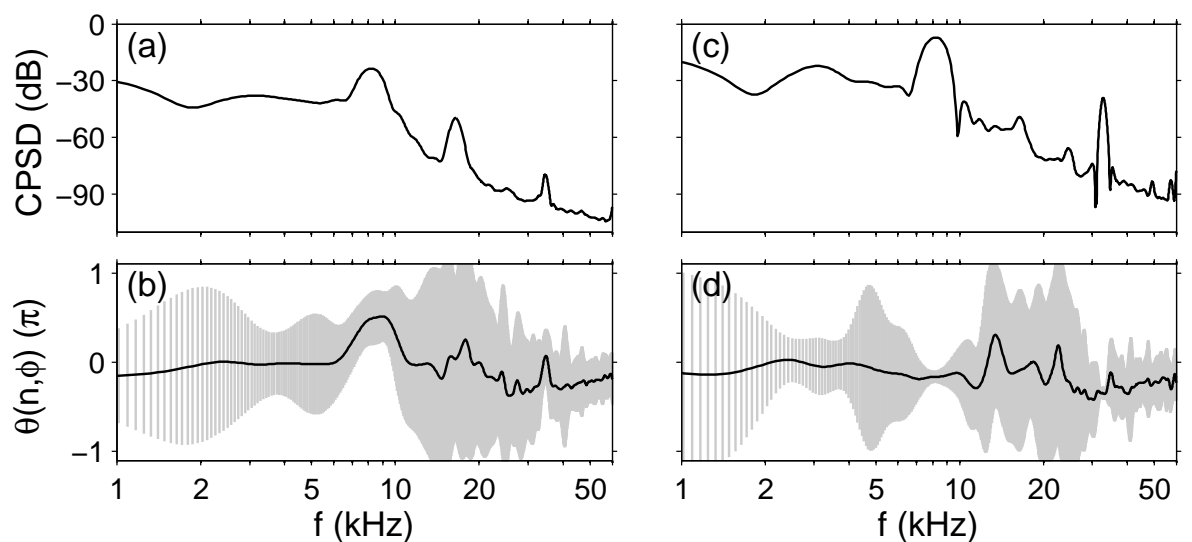

Figure 10. Cross-power spectra and cross-phase spectra between plasma density and plasma potential fluctuations for weakly developed turbulence $(\mathrm{a}, \mathrm{b})$, and controlled turbulence by the co-rotating exciter $(\mathrm{c}, \mathrm{d})$. The grey error bars indicate the statistical confidence. 
In uncontrolled turbulence the phase in the range $f=7-10 \mathrm{kHz}$ of the broadened spectral peak is $\theta \approx \pi / 2$, which is in agreement with earlier experimental results [29]. In controlled turbulence [Fig. 10(c-d)] the spectral peak increases in amplitude and the phase is significantly reduced to approximately $\theta \approx 0$. Consequently the cross-field transport, which is proportional to $\tilde{n} \tilde{E} \sin (\theta)$, is strongly reduced.

\subsection{Electromagnetic synchronization}

Figure 11 shows the frequency spectrum and the space-time diagram of a coherent $m_{0}=2$ drift wave mode with a frequency $f_{0}=6.8 \mathrm{kHz}$. The smaller frequency peaks originate from a coupling with a second, weakly developed drift wave mode. The spacetime evolution of density fluctuations is shown in Fig. 11(b).
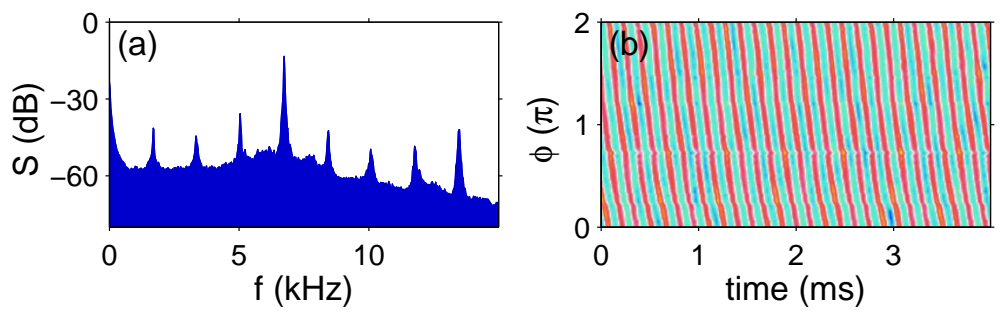

Figure 11. (Color online) Frequency spectrum (a) and space-time diagram (b) of a coherent $m_{0}=2$ drift wave mode.

The drift wave is controlled with a co-rotating electromagnetic exciter signal and the result of the frequency scan is shown in Fig. 12. The exciter signal is a $m_{d}=2$ mode with an induced plasma current density of $j_{d}=74 \mathrm{~mA} / \mathrm{cm}^{2}$. The spectrum of the controlled drift wave is colored in red, the spectrum of the uncontrolled drift wave is superimposed in blue, and the exciter frequency is indicated in green. In the first three diagrams the exciter approaches the drift wave frequency. During the scan, sidebands occur, corresponding to the sum and difference between exciter and drift wave frequency (see above). Pronounced periodic pulling occurs in Figs. 12(b,c), evident by the characteristic triangular shape of the sidebands around the drift wave peak. In Fig. 12(d) the exciter and drift wave frequency are almost equal. The sideband structure disappears and the drift wave locks to the exciter signal at a frequency $f_{d} \approx f_{0}=6.75 \mathrm{kHz}$. The exciter signal pulls the drift wave up to a frequency of $f=6.95 \mathrm{kHz}$ [Figs. 12(e-g)] until the drift wave unlocks at $f=7 \mathrm{kHz}$. In diagrams $12(\mathrm{~h}, \mathrm{i})$ the drift wave peak and the exciter frequency are well separated and the drift wave frequency is the same as the unperturbed drift wave in Fig. 12(a).

We inspect Figs. 12(b,c) closer, where the exciter frequency is near the drift wave frequency. Due to the nonlinear interaction between the exciter signal and the drift wave, a dense arrangement of sidebands occurs on each side of the drift wave peak. If the exciter frequency gets very close to the drift wave frequency, the sideband structure becomes asymmetric. Here periodic pulling, i.e., incomplete synchronization, occurs 

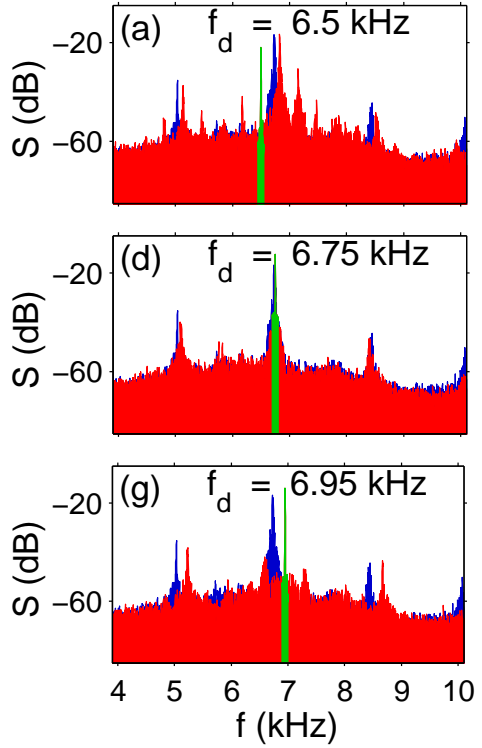
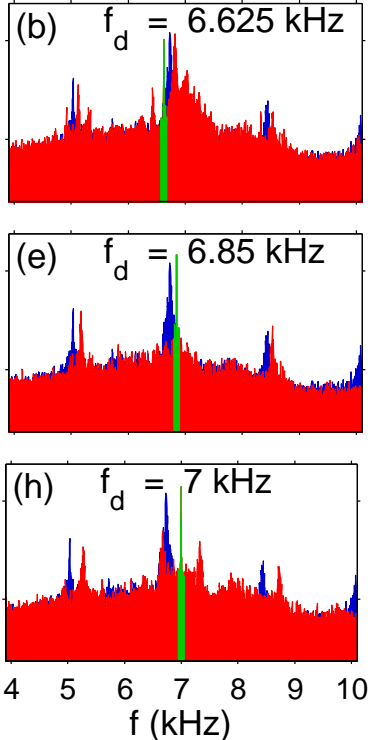
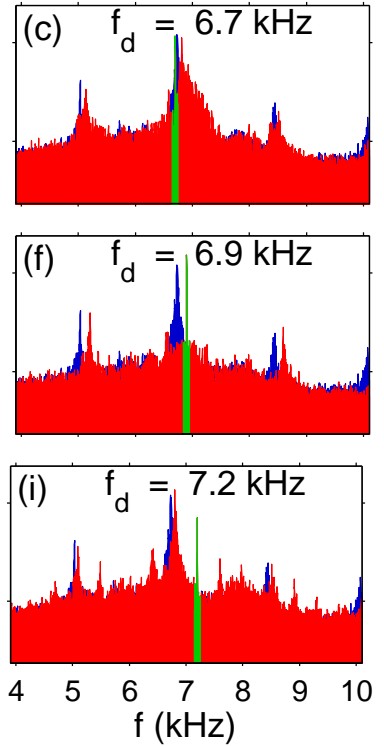

Figure 12. (Color online) Synchronization of a coherent $m_{0}=2$ drift wave mode using the electromagnetic exciter. A chosen sequence of spectra is shown where the exciter frequency is scanned from $6.5 \mathrm{kHz}$ to $7.2 \mathrm{kHz}$ for several plasma discharges. The spectrum of the unperturbed coherent drift wave is shown in blue in the background. The exciter frequency is marked in green.

[30]. The peak develops a typical triangular shape with a longer "tail" towards higher frequencies. The sequence of frequency spectra in the Figs. 13(a-d) shows in detail how the drift wave spectrum changes when the exciter signal approaches the drift wave frequency. The time trace of the phase difference between drift wave and exciter for each spectrum is plotted in Fig. 13(e). In Fig. 13(a) the exciter frequency is smaller
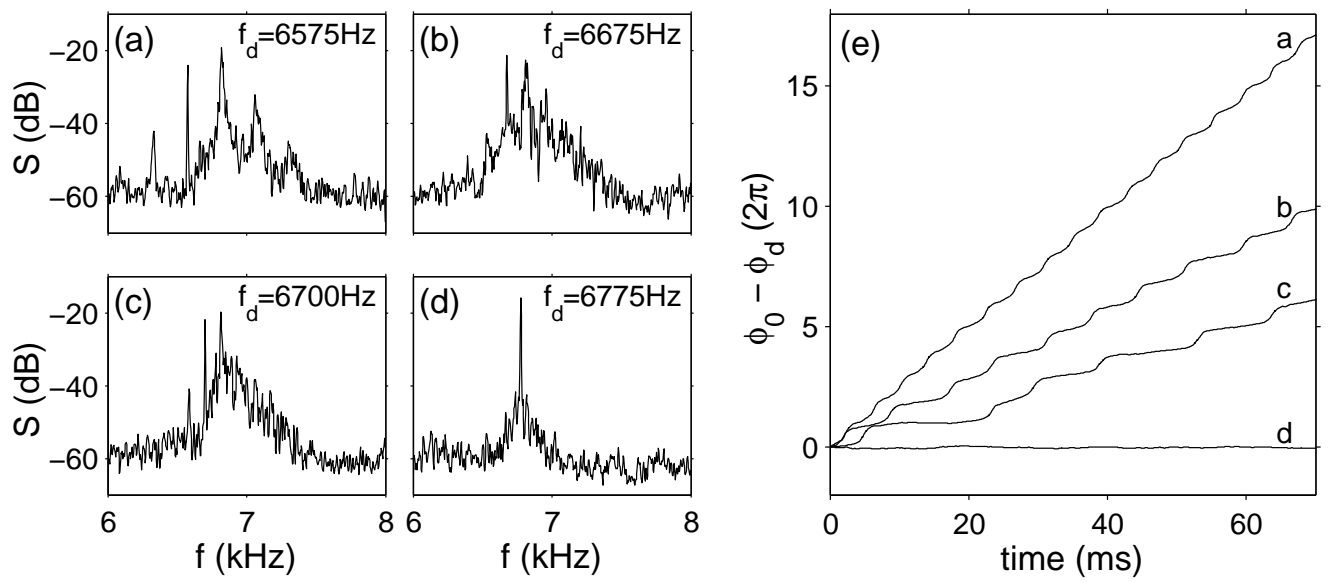

Figure 13. Periodic pulling of a coherent drift wave with active co-rotating electromagnetic exciter: (a-d) frequency spectra in the vicinity of the drift wave $\left(f_{0}=6.8 \mathrm{kHz}\right),(\mathrm{e})$ time series of the phase difference between exciter signal, and drift wave signal for the cases (a-d). 
than the drift wave frequency $\Delta f=f_{d}-f_{0}=-325 \mathrm{~Hz}$. Sidebands corresponding to the frequencies $f_{0} \pm n\left(f_{d}-f_{0}\right)$ arise. The phase difference increases as a steep staircase [Fig. 13(e) graph a]. In Fig. 13(b) the exciter frequency is closer to the drift wave $(\Delta f=-225 \mathrm{~Hz})$. A pronounced tail of sidebands is found towards higher frequencies above the drift wave peak. The slope of the phase difference trace is less steep while the width of the steps is increased. For $\Delta f=-100 \mathrm{~Hz}$ the spectrum shows a pronounced triangular shape of sidebands around the drift wave frequency, while the slope of the phase difference trace is further decreased and the step width is further increased. The spectrum in Fig. 13(d) shows the locked drift wave for a mismatch $\Delta f=-25 \mathrm{~Hz}$. No sidebands are observed, the width of the spectral peak is significantly decreased, and the phase difference stays constant. Similar frequency spectra of periodic pulling have been found for the control of drift waves using the electrostatic exciter setup [cf. Fig. $6(\mathrm{c})$ and [28]].

\section{Summary and conclusions}

The control of single coherent drift modes and drift wave turbulence has been experimentally studied in the linear magnetized laboratory device VINETA. The drift wave instability leads to density and potential fluctuations that propagate azimuthally into the electron diamagnetic drift direction. Coherent drift waves occur as azimuthally rotating eigenmode structures. The tight coupling between the drifts perpendicular to the magnetic field and the parallel electron currents is the reason for the threedimensional nature of drift waves. Two different spatiotemporal open-loop control systems, one electrostatic and one electromagnetic, consisting of azimuthal octupole arrangements of electrodes and magnetic saddle coils, respectively, are applied to influence the drift wave dynamics.

The electrodes of the electrostatic exciter have a direct electric contact to the plasma and parallel current and potential perturbations can be driven. The electromagnetic exciter drives parallel plasma currents as well but without electrical contact to the plasma by electromagnetic induction. The drive of mode selective parallel plasma currents turns out to be crucial for the control of the drift waves. This is strongly supported by the fact, that the electrostatic exciter achieves no influence at all if operated with electrically insulated electrodes (to suppress parallel plasma currents). In addition, 2D drift wave simulations have shown only controlled states if a rotating current pattern with a suitable mode structure is applied [16].

It is important to distinguish between the perturbation created with the exciter, actual drift wave fluctuations, and controlled drift waves. The exciter driven parallel plasma currents $\tilde{j}_{\|}$result in potential perturbations $\tilde{\phi}$ along the flux tubes of the entire plasma column driving $E \times B$-drifts with

$$
\tilde{j}_{\|} \rightarrow \tilde{\phi} \rightarrow v_{E \times B} \rightarrow \tilde{n}
$$

Thus, the driven density perturbations $\tilde{n}$ are phase shifted by $\pi / 2$ with respect to the potential perturbations $\tilde{\phi}$. However, in the case of a synchronized coherent drift wave, 
the perturbations excited by the control signal couple nonlinearly with the density and potential fluctuations of the drift wave and synchronize them without changing the drift waves cross-phase between density and potential fluctuations of typically $\theta \approx 0$. This finding clearly shows that the drift wave fluctuation is not only suppressed by the large density perturbation produced by the exciter but that indeed synchronization of the drift wave is achieved and the fundamental drift wave properties as e.g. the small phase shift $\theta \approx 0$ remain essentially conserved.

For both control systems, the local perturbation of the plasma currents lead to global control of the drift wave dynamics by nonlinear coupling to the parallel electron dynamics. Synchronization effects of coherent drift waves are observed if the driver's mode pattern matches exactly the one of the drift mode. If the driver frequency approaches the drift wave frequency in a frequency scan, incomplete synchronization is first observed. This nonlinear effect is apparent by a characteristic triangular shape of the frequency spectrum and by phase slippage. If the driver frequency is close to the drift wave frequency, the drift wave locks to the spatiotemporal control signal and frequency pulling occurs.

Experiments with the electrostatic exciter show that synchronization of drift wave turbulence to establish a pre-selected mode pattern can be achieved. The turbulent dynamics is reduced to a coherent drift mode with a well defined frequency and mode number, though with a (suppressed) broadband turbulent background. The amplitude of the control signal must exceed a certain value before synchronization occurs. Control of drift wave turbulence can be achieved only if the propagation direction of the control signal coincides with the propagation direction of self-excited coherent drift waves. This is in agreement with previous reports $[16,28,31]$. The cross-phase between density and potential fluctuations changes from $\theta \approx \pi / 2$ in turbulence to $\theta \approx 0$ in the controlled case. Consequently, although the density fluctuation amplitude is not decreased, the reduced phase shift will lead to considerable reduction of the fluctuation driven crossfield particle transport. The turbulent regime is forced into a coherent regime by the nonlinear coupling with the exciter signal. Coherent drift waves - also the synchronized ones - have a cross-phase close to zero. However, the transport aspects can only be addressed in a meaningful way in a confinement experiment.

\section{References}

[1] Lehnert B 1958 Nature 181 331-332

[2] Hoh F C and Lehnert B 1960 Phys. Fluids 3 600-607

[3] Chu T K, Hendel H W and Politzer P A 1967 Phys. Rev. Lett. 19 1110-1113

[4] Kadomtsev B B and Nedospasov A V 1960 J. Nucl. Energy, C: Plasma Phys. 1 230-235

[5] Zweben S J and Gould R W 1985 Nucl. Fusion 25 171-183

[6] Horton W 1999 Rev. Mod. Phys. 71 735-778

[7] Balescu R 2003 Phys. Rev. Lett. 68 046409(13)

[8] Keen B E and Aldridge R V 1971 J. Phys. A 4 120-141

[9] Odajima K, Nishida Y and Hatta Y 1974 Phys. Fluids 17 1631-1633 
[10] Uckan T, Richards B, Bengtson R, Carreras B, Li G, Hurwitz P, Rowan W, Tsui H and Wootton A 1995 Nucl. Fusion 35 487-494

[11] Xu Y, Weynants R R, Jachmich S, Van Schoor M, Vergote M, Peleman P, Jakubowski M W, Mitri M, Reiser D, Unterberg B and Finken K H (TEXTOR team) 2006 Phys. Rev. Lett. 97165003 (pages 4)

[12] Foster M, McCool S and Wootton A 1995 Nucl. Fusion 35 329-333

[13] Ghendrih P, Becoulet M, Colas L, Grosman A, Guirlet R, Gunn J, Loarer T, Azeroual A, Basiuk V, Beaumont B, Becoulet A, Beyer P, Bremond S, Bucalossi J, Capes H, Corre Y, Costanzo L, Michelis C D, Devynck P, Feron S, Friant C, Garbet X, Giannella R, Grisolia C, Hess W, Hogan J, Ladurelle L, Laugier F, Martin G, Mattioli M, Meslin B, Monier-Garbet P, Moulin D, Nguyen F, Pascal J Y, Pecquet A L, Pegourie B, Reichle R, Saint-Laurent F, Vallet J C, Zabiego M and Team T S 2002 Nucl. Fusion 42 1221-1250

[14] Boedo J, Rudakov D, Hollmann E, Moyer R, McKee G, Burrell K, Evans T, Leonard A, West W, Fenstermacher M, Groth M, Allen S, Zeng L, Wang G and Watkins J 2005 J. Nucl. Mater. 337-339 771-775

[15] Tokar M Z, Evans T E, Gupta A, Singh R, Kaw P and Wolf R C 2007 Phys. Rev. Lett. 98 095001-4

[16] Schröder C, Klinger T, Block D, Piel A, Bonhomme G and Naulin V 2001 Phys. Rev. Lett. 86 $5711-5714$

[17] Block D, Schröder C, Klinger T and Piel A 2001 Contrib. Plasma Phys. 41 455-460

[18] Brochard F, Gravier E and Bonhomme G 2006 Phys. Rev. Lett. 73 036403(7)

[19] Kauschke U 1992 Plasma Phys. Control. Fusion 34 1303-1337

[20] Grulke O, Franck C M, Klinger T, Schröder C, Stark A, Windisch T and Zalach J 2005 Contrib. Plasma Phys. 45 385-395

[21] Franck C M, Grulke O and Klinger T 2003 Phys. Plasmas 10 323-325

[22] Latten A, Klinger T, Piel A and Pierre T 1995 Rev. Sci. Instrum. 66 3254-3262

[23] Schröder C, Grulke O, Klinger T and Naulin V 2005 Phys. Plasmas 12 042103(6)

[24] Schröder C, Grulke O, Klinger T and Naulin V 2004 Phys. Plasmas 11 4249-4253

[25] Chen F F 1965 Phys. Fluids 8 912-919

[26] Ullrich S 2010 Electromagnetic drift waves, Ph.D. thesis, University of Greifswald

[27] Koepke M E, Dinklage A, Klinger T and Wilke C 2001 Phys. Plasmas 8 1432-1436

[28] Block D, Piel A, Schröder C and Klinger T 2001 Phys. Rev. E 63 0564011(7)

[29] Windisch T, Grulke O and Klinger T 2006 Phys. Scripta T122 15-18

[30] Koepke M E and Hartley D M 1991 Phys. Rev. A 44 6877-6887

[31] Brochard F, Bonhomme G, Gravier E, Oldenbürger S and Philipp M 2006 Phys. Plasmas 13 052509-9 